\title{
CSI-SF: Estimating Wireless Channel State Using CSI Sampling \& Fusion
}

\author{
Riccardo Crepaldi*, Jeongkeun Lee ${ }^{\dagger}$, Raul Etkin ${ }^{\dagger}$, Sung-Ju Lee ${ }^{\dagger}$, Robin Kravets* \\ *University of Illinois at Urbana-Champaign \\ ${ }^{\dagger}$ Hewlett-Packard Laboratories \\ Email: $\{$ rcrepal2,rhk\}@illinois.edu, \{jklee,raul.etkin,sjlee\}@hp.com
}

\begin{abstract}
One of the key features of high speed WLAN such as 802.11n is the use of MIMO (Multiple Input Multiple Output) antenna technology. The MIMO channel is described with fine granularity by Channel State Information (CSI) that can be utilized in various ways to maximize the network performance. Many complex parameters of a MIMO system require numerous samples to obtain CSI for all possible channel configurations. As a result, measuring the complete CSI space requires excessive sampling overhead and thus degrades the network performance. We propose CSI-SF (CSI with Sampling \& Fusion), a method for estimating CSI using a small number of frame transmissions and extrapolating data to settings that have not been sampled. For instance, we predict CSI of multiple stream settings using CSI obtained only from single stream packets. We evaluate the effectiveness of CSI-SF on various network scenarios using our 802.11n testbed and show that CSI-SF provides an accurate, complete knowledge of the MIMO channel with reduced overhead from traditional sampling. We also show that CSI-SF can be applied to network algorithms such as rate adaptation, antenna selection and association control to significantly improve their performance and efficiency.
\end{abstract}

The new IEEE 802.11n [1] and the emerging IEEE 802.11ac [2] standards aim to provide very high throughput WLAN to meet this growing demand of applications and services. Some of the key enhancements used for increasing the throughput are using wider, bonded channels $(40 \mathrm{MHz}$ in $802.11 \mathrm{n}$ and up to $160 \mathrm{MHz}$ in $802.11 \mathrm{ac}$ ), frame aggregation and block acknowledgments, a short guard interval, and MIMO (Multiple Input Multiple Output) antennas [3], [4]. MIMO is a popular technology in wireless communications (e.g., 802.11n, WiMax, 3GPP LTE, etc.) to increase link throughput and distance. 802.11n devices in the current market support up to three MIMO spatial streams.

Algorithms and protocols for WLAN need to consider the new features offered by multiple antennas; for instance, rate adaptation is not only selecting modulation and coding rate but also the number of concurrent spatial data streams transmitted. In order to achieve optimal WLAN performance, we require a detailed knowledge of the wireless link, which can be acquired through the Channel State Information (CSI). CSI represents the current condition of the channel, and consists of the attenuation and phase shift experienced by each spatial stream to each receive antenna in each of the OFDM subcarriers. CSI is provided in the $802.11 \mathrm{n}$ hardware by analyzing received packets using training sequences in the packet headers. For network algorithms such as rate selection, AP association, channel assignment, etc., to make a timely, optimal decision, accurate CSI estimates under various settings (e.g., different number of spatial streams, transmission antennas used, transmission powers, etc.) must be known. However, some of these settings might not have been sampled in recently received packets and additional frame transmissions are required to obtain the complete CSI. This extra process consumes bandwidth and increases latency, and hence such unnecessary sampling should be avoided.

We present CSI-SF (Channel State Information with Sampling and Fusion), a CSI processing technique that predicts the complete CSI, including those of non-sampled MIMO configurations, using a small number of samples. For example, with CSI-SF we can predict the CSI of a $3 \times 3$ channel using CSI measured from packets sent using a $1 \times 3$ configuration.

Many implementations of 802.11 n require successful decoding of a data packet in order to obtain the CSI.

In current CSI estimation approaches, obtaining CSI for all possible configurations of a $3 \times 3$ system requires seven samples: 1-stream Modulation and Coding Schemes (MCS) require three probes, one per each TX antenna. Similarly, 2-stream MCSs require three probes to collect CSI for each combination of two antennas. Lastly, 3-stream MCSs require a single probe using a transmission from all three transmit antennas. The number of required samples increases dramatically when the system supports $4 \times 4$ communication or larger channel widths. Moreover, hardware commonly provides CSI reports only for unicast packets, thus limiting the possibility of opportunistically collecting the CSI matrices by eavesdropping; a node must be connected to an access point to actively send or receive probing packets.

We address the problem of estimating $p \times q \times R$ CSI data structures using packets encoded with $m_{i} \times n_{i} \times W_{i}$ schemes, where $p \geq m_{i}, q \geq n_{i}, R \geq W_{i}$, and $(p, q, R) \neq\left(m_{i}, n_{i}, W_{i}\right)$, where $i$ is the packet index. That is, we use CSI data structures obtained from multiple packet transmissions to estimate larger CSI data structures. We describe this problem in the context of an $802.11 \mathrm{n}$ system. However, similar ideas can be applied to CSI estimation in other MIMO OFDM systems (e.g. WiMAX).

We aggregate CSI obtained from multiple packets to derive CSI corresponding to a larger channel than those used to send and receive these packets. For example, a $2 \times 2 \times 56$ CSI matrix can be obtained by combining the CSI information that are derived from two packets transmitted using a $2 \times 1 \times 56$ 
configuration, as long as different transmit antennas are used to send the two packets. The combining of CSI from multiple packets can be used to:

- estimate a $m \times q \times W$ CSI matrix using packets sent/received with an $m \times n \times W$ configuration, with $q>n$ (i.e., estimate a CSI matrix corresponding to more transmit antennas than the number used to send each packets for estimation);

- estimate a $m \times n \times R$ CSI matrix using packets sent/received with an $m \times n \times W$ configuration with $R>W$ (i.e. estimate a CSI matrix corresponding to a larger bandwidth than the one used to send each packets for estimation);

- estimate a CSI matrix combining the above two cases (i.e. enlarge the number of antennas and bandwidth in the combined CSI).

When combining CSI, three aspects of the MIMO transmission chain must be considered. First, the 802.11n standard allows the use of a precoding matrix $Q$ to map the bit streams into the channel. Typically, the precoding matrix $Q$ does not need to be known at the receiver, and the channel estimation algorithm provides an estimate which includes its effect. However, the CSI-SF combining technique requires the receiver to know $Q$ to isolate the channel information from the CSI report.

Secondly, due to regulations and practical limitations, there is a total power constraint for the transmitted signal. In order to meet the total power constraint, the power transmitted on each subcarrier may vary for different configurations with different bandwidths or number of transmit antennas.

Finally, in most WLAN deployments, dynamic transmission power is used in combination with rate control to reduce power consumption. Commodity hardware allows the user to choose a transmission power level. If the transmitted power closely follows the level chosen by the user, we can apply a scale factor to the CSI of a packet transmitted at a specific power level to estimate the CSI of a different power level. For example, if we receive a packet sent at $7 \mathrm{dBm}$ and we are interested in the estimated CSI for a transmission with the same antenna configuration at $5 \mathrm{dBm}$, it is enough to subtract $2 \mathrm{~dB}$ from the magnitude of the original CSI to obtain the new one. However, practical limitations influence the power control capabilities of real transceivers. Power amplifiers are not perfectly linear, producing increasing distortion as they are driven closer to their maximum rated power. The distortion introduced by the transmitter amplifiers has a bigger impact on MCSs with larger coding rates and higher order modulations. As a result, many transceivers limit the output power used for high rate MCSs through various power caps.

In our poster we will discuss how CSI-SF addresses these three issues as well as the impact of introducing approximation required when some hardware-specific information is not known or to limit the overhead of our solution.

\section{EVALUATION}

We evaluate the performance of CSI-SF using CSI information collected on our 802.11n testbed. We deployed five

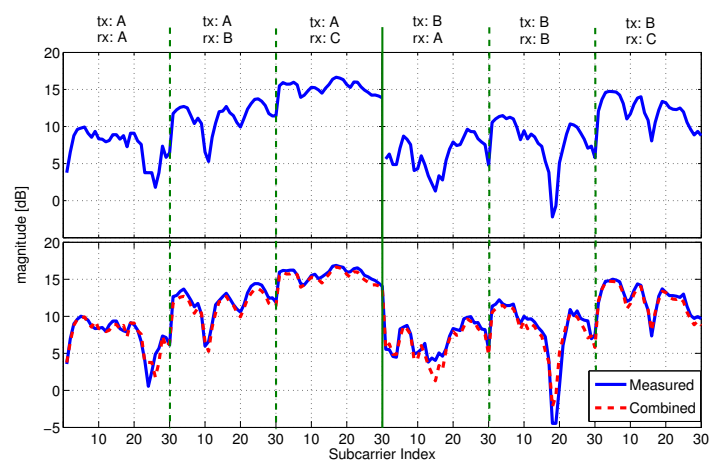

Fig. 1. Magnitude of each CSI element of two single stream packets (top) and their dual stream combination, compared with an actual dual stream packet (bottom).

nodes, equipped with an Intel 5300 chipset that supports up to $3 \times 3$ MIMO transmission. Modified chipset firmware and kernel from [5] are used to obtain the CSI matrices of successfully received 802.11n data frames and transfer the matrices to userspace for further analysis. We deployed the nodes in a cubicle office environment and collected CSI for different links, power settings and antenna configurations.

In Figure 1, we plot the magnitude of each element of the CSI matrices of two 1-stream packets (top) and compare the combined CSI obtained using CSI-SF with the actual 2-stream CSI (bottom). The combined and actual CSI are only slightly different probably due to small channel variations between the time at which the three packets were sampled. However, the difference is small, and when CSI is used to compute aggregate metrics such as eSNR, the difference becomes negligible as we show in the following subsections.

While a complete characterization of a given channel by looking at every element of its CSI matrix might be useful at times, in most applications (e.g., bitrate adaptation) an aggregate metric obtained from the CSI is sufficient. Hence we use the effective $S N R$ (eSNR) value, as described in [6], to evaluate the efficacy of CSI-SF. eSNR first computes the SNR and bit-error rate (BER) of each OFDM subcarrier. From the subcarrier-specific information, a channel-wide BER is computed and translated into eSNR. The efficacy of eSNR in terms of accurately metricizing the quality of OFDM MIMO channel compared with the per-packet SNR metric that 802.11 drivers usually provide in the form of Received (or Relative) Signal Strength Indicator (RSSI) ${ }^{1}$ is shown in [6]. In the rest of this section, we use the term, SNR, to indicate the per-packet SNR directly given by drivers.

We evaluate the accuracy of CSI-SF by comparing the eSNR obtained from the real CSI information against eSNR obtained using CSI-SF, which we call estimated effective SNR ( $\left.{ }^{2} \mathrm{SNR}\right)$. Figures 2 highlights the relation between each metric (SNR, eSNR and $\mathrm{e}^{2} \mathrm{SNR}$ ) and the maximum supported rate on $2 \times 3$ and on $3 \times 3$ links we tested on various environments and

\footnotetext{
${ }^{1}$ Receivers with multiple RX antennas usually compute a packet RSSI by averaging or taking the maximum of RSSIs measured for each RX antenna.
} 

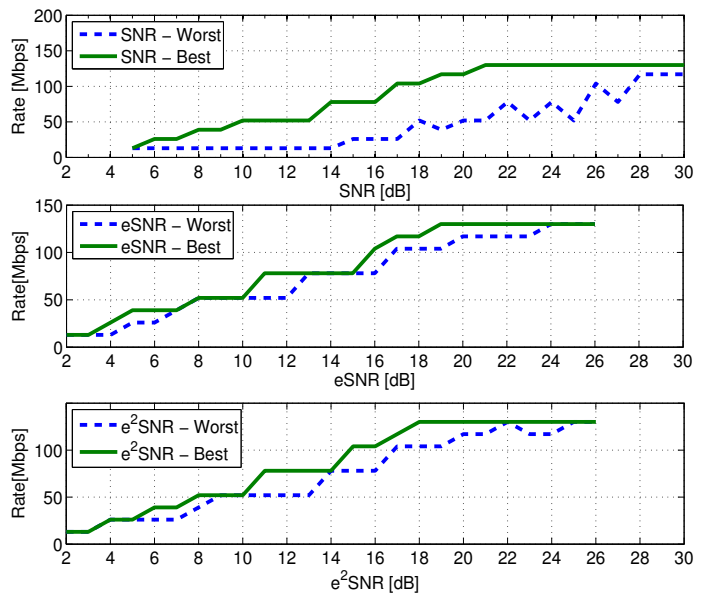
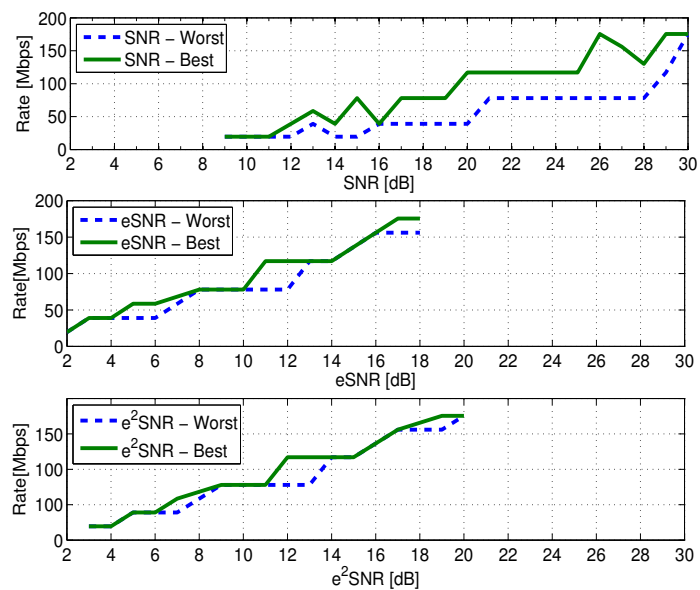

Fig. 2. Link rates (Mbps, y-axis) for the best and the worst links as a function of various metrics (SNR, eSNRand $\mathrm{e}^{2} \mathrm{SNR}$ ) for 2-stream (left) and 3-stream (right) MCSs using a $20 \mathrm{MHz}$, channel.

configurations. We say a bitrate is "supported" by a given link when the packet error rate for the MCS at that bitrate is measured to be smaller than $10 \%$. We define "link rate" the maximum supported bitrate for a link. For rate adaptation algorithms and similar applications, it is imperative that the link quality metric shows a predictable behavior. However, for each metric, links with the same metric value can provide a different link rate. For each value of SNR, eSNR and $\mathrm{e}^{2} \mathrm{SNR}$ we find the link with the highest and the lowest link rate and denote them as the best and the worst links, respectively. For each metric we plot the link rate for the best and the worst link. A good metric will exhibit two properties:

- a small gap between the best and the worst links (ideally the two lines should overlap) to enable an accurate prediction of the link rate;

- a monotonic relation between the metric value and the link rate, so that we can always consider a higher metric value as a better link quality.

The use of packet SNR (the topmost graphs) results in significant gaps between the bitrates supported by the best and the worst links with the same SNR value. A rate selection algorithm based on SNR could choose to be conservative and select a low bitrate, and then increase the rate until the link rate is reached, or be optimistic and start from the bitrate supported by the best link with the same SNR value and then fallback to a lower rate if the selected one is not supported. In both cases the algorithm takes time to converge to the optimal rate. The bottom part of the figures show the results of using eSNR and $\mathrm{e}^{2} \mathrm{SNR}$ metrics. For the most part, the best and the worst link overlap or show a small gap limited to one MCS difference. Most of all, $e^{2} S N R$ from combined CSI exhibits a promising bitrate predictability, which is comparable to that of eSNR of actual CSI.

CSI-SF not only can be used for estimating a MIMO configuration with a larger number of antennas, but also for a channel that is bonding two or more narrower channel which have been sampled independently. Bonding of two adjacent channel is enforced in $802.11 \mathrm{n}$ to increase the bandwidth, and the new 802.11ac standard allows bonding of non-adjacent channels up to a bandwidth of $160 \mathrm{MHz}$. We tested the accuracy of CSI-SF on bonded channels by comparing the $\mathrm{e}^{2} \mathrm{SNR}$ obtained from combining two $20 \mathrm{MHz}$ link sent on different channels, and the actual eSNR of packets sent on the bonded channel, on several different links. The maximum value for the standard deviation is lower than $2 d B$. The distance between the synthetic $\mathrm{e}^{2} \mathrm{SNR}$ and the real eSNR can be explained with the large time between the reception of packets on the different channels in our experiments, due to hardware configuration constraints.

\section{CONCLUSION}

In this poster abstract we present CSI-SF, a method for estimating CSI using a small number of frame transmissions and extrapolating data to settings that have not been sampled. Our preliminary results, based on the CSI collected in our 802.11n network testbed, show that CSI-SF can be utilized not only for estimating CSI for larger number of streams, but also for wider channels. We believe the algorithm designers for rate adaptation, beamforming, association control can take advantage of CSI-SF to improve their performance. We also argue that CSI-SF will be even more beneficial in future networks with more antenna configurations and wider bonded channels.

\section{REFERENCES}

[1] "IEEE 802.11n-2009 Amendment 5: Enhancements for Higher Throughput," IEEE-SA. 29 October 2009.

[2] "IEEE 802.11ac: Very High Throughput $<6$ GHz," IEEE Standards.

[3] A. J. Paulraj, D. A. Gore, R. U. Nabar, and H. Bolcskei, "An overview of MIMO communications - a key to gigabit wireless," Proceedings of IEEE, 2004.

[4] A. Goldsmith, S. A. Jafar, N. Jindal, and S. Vishwanath, "Capacity limits of MIMO channels," IEEE Journal on Selected Areas in Communications, 2003.

[5] D. Halperin et al., "802.11n CSI Tool based on iwlwifi and Linux-2.6," https://github.com/dhalperi/linux-80211n-csitool-supplementary

[6] D. Halperin, W. Hu, A. Sheth, and D. Wetherall, "Predictable 802.11 packet delivery from wireless channel measurements," in Proc. of ACM SIGCOMM, 2010. 